\title{
Human Endogenous Retrovirus-H Long Terminal Repeat- Associating Protein 2 (HHLA2) is a Novel Immune Checkpoint Protein in Lung Cancer which Predicts Survival
}

\author{
Mayada Saad Farrag1*, Eman Mohamad Ibrahim², Tamer A El-Hadidy ${ }^{3}$, \\ Mohamed Farouk Akl ${ }^{4}$, Alyaa R Elsergany ${ }^{5}$, Heba Wagih Abdelwahab ${ }^{3}$
}

\begin{abstract}
Background: Lung cancer is one of the most frequently diagnosed malignancies. Human endogenous retrovirus- $\mathrm{H}$ long terminal repeat-associating protein 2 (HHLA2) is a recently discovered ligand of the B7 family. Blocking this immune checkpoint has become an important treatment option for lung cancer. Methods: The study includes 62 biopsy specimens either bronchoscopic or CT-guided biopsies diagnosed as lung cancer in Hospitals of Faculty of Medicine, Mansoura University, Egypt during the period from 2016 to 2020. Immunohistochemical Staining for HHLA2 and EGFR was performed. HHLA2 expression was assessed in different pathological types of lung Cancer, and it was correlated with other clinicopathologic parameters and patient prognosis. Results: We found a significant association between HHLA2 expression and metastasis. About $83 \%$ of patients presented with metastasis showed positive expression of HHLA2 compared to $44.4 \%$ in patients with no metastasis $(\mathrm{p}=0.02)$. Also, results show significant mild positive correlation between expression of HHLA2 and EGFR markers ( $\mathrm{p}=0.045)$. The mean OS time in cases with positive HHLA2 expression was nearly half that of patients with negative expression of the markers. However, this difference was not statistically significant. But, PFS of patients was significantly lower among the group with positive expression of HHLA2 compared to the group with negative expression of HHLA2 ( $\mathrm{p}=0.01)$. Conclusions: This study reports that recently discovered, HHLA2 is over expressed in lung cancer associating with higher stage. It is also correlated with EGFR overexpression. HHLA2 could serve as a predictor of progression and distant metastasis. Also, it has potential to be effective immune target in lung cancer immunotherapy such as checkpoint blockade and antibody-drug conjugate treatment.
\end{abstract}

Keywords: HHLA2- Immunotherapy- Lung cancer- Metastasis- PFS

Asian Pac J Cancer Prev, 22 (6), 1883-1889

\section{Introduction}

Lung cancer is one of the most frequently diagnosed malignancies. It is the major cause of cancer related mortality in males and the second major cause of cancer related mortality in females all over the world (Torre et al., 2016). In Egypt, National Population-Based Cancer Registry Program 2008 - 2011 ranked lung cancer as the sixth most common cancer in both genders (Ibrahim et al., 2014). Pathological review of lung cancer tissues is fundamental in diagnosis, prognosis and treatment decision of these cancers. Recent advances in genomic profiling identify new targetable oncogenes in lung cancer. Understanding the heterogeneous tumor microenvironment can open new avenues for better management (Chen et al., 2014).

The value of immunotherapy is recently emphasized in different tumors (Chang et al., 2019). Blocking the immune checkpoint (ICP) has become an important treatment option in the management of lung cancer. Programmed death ligand-1 (PDL-1) inhibitors are the first used ICP inhibitor. They have been approved in the United States and Europe as second-line therapy for metastatic NSCLCs (Zago et al., 2016). However, there are challenges to immunotherapy including immune-related toxicity and resistance to immunotherapy. This would cause more difficulties on tailoring treatment protocols (Zhang et al., 2019).

Human endogenous retrovirus-H long terminal repeat-associating protein 2 (HHLA2) is a recently

${ }^{I}$ Department of Pathology, Faculty of Medicine, Port Said University, Port Said, Egypt. ${ }^{2}$ Department of Pathology, Faculty of Medicine, Mansoura University, Mansoura, Egypt. ${ }^{3}$ Department Chest Medicine, Faculty of Medicine, Mansoura University, Mansoura, Egypt. ${ }^{4}$ Department of Clinical Oncology \& Nuclear Medicine, Faculty of Medicine, Mansoura University, Mansoura, Egypt. ${ }^{5}$ Department Internal Medicine, Oncology Center, Faculty of Medicine, Mansoura University, Mansoura, Egypt. *For Correspondence: dr.midosaad@yahoo.com 
discovered ligand of the B7 family, and was first described as a $\mathrm{T}$ cell co-inhibitory molecule (Cheng et al., 2017). It belongs to group III of the B7 family (Yan et al., 2019). The current study assesses HHLA2 immunohistochemical expression lung Cancer. It differentiates between HHLA2 expressions in different histopathological types. It also correlates the expression with other different clinicopathologic parameters. Thus, we can study HHLA2 impact on patient prognosis. It also could help clarify the value of $H H L A 2$ as potential target for immunotherapy in lung cancer.

\section{Materials and Methods}

The current study is retrospective study. It includes 62 biopsy specimens either bronchoscopic or CT-guided biopsies diagnosed as lung cancer in Oncology Center and the Clinical Oncology and Nuclear Medicine department, in collaboration with the Chest Medicine and Pathology departments, Mansoura University, Mansoura, Egypt during the period from 2016 to 2020. This work had the approval of institutional research board of Faculty of Medicine, Mansoura University (R.20.09.1026).

The cases were chosen randomly. Simple random sampling was performed using excel software to choose the final sample. Any chosen patient with absent paraffin block was replaced by another using the same method. The relevant clinicopathologic data was collected. Also, we followed the clinical outcome of the patients in the form of progression free survival (PFS) and overall survival (OS). Overall survival was calculated from diagnosis to death. PFS is the time from treatment initiation to 1 st recurrence or metastasis or death. H\&E slides of the pathologic specimens were reviewed to assess adequacy of tumor tissue for immunostaining.

Immunohistochemical Staining: Sections from formalin-fixed paraffin-embedded tissue blocks were deparaffinized and hydrated by standard procedures. Antibodies for HHLA2 (Rabbit polyclonal Ab, A13262, IgG, Abclonal, Inc) and EGFR (Rabbit polyclonal Ab, A11351 IgG, Abclonal, Inc) were utilized based on manufacturer instructions with appropriate positive control and negative control.

Immunohistochemical Assessment: slides were independently scored by 2 pathologists who had no knowledge about patients' data. Positive HHLA2 expression was defined as $>5 \%$ positive tumor cells (Yan et al., 2019). The intensity of HHLA2 staining was recorded as $0,1,2$ and 3 for absent, mild, moderate and strong expression, respectively (Cheng et al., 2018). Positive EGFR expression was considered when staining $>1 \%$ tumor cells (Petersen et al., 2017).

Statistical Analysis: Data was analyzed by SPSS software V.26. Categorical data was expressed as frequencies and percentages. Continuous data was shown as mean (SD) or Median (minimum-maximum) based on Shapiro-Wilk testing for the assumption of normal data distribution. Statistical significance testing of continuous data was done using Mann-Whitney U or Median Test. while, chi square test or Fisher's Exact Test were used for categorical data, wherever appropriate. Spearman's rho test was utilized to explore the correlation between HHLA2 and EGFR markers. Kaplan-Meier test was utilized to assess OS and PFS of patients as regards tumor expression of HHLA2. Comparison was performed utilizing Log Rank (Mantel-Cox). Level of significance level was at 0.05.

\section{Results}

\section{Clinicopathological Characteristics of the Patients}

The study enrolled 62 lung carcinoma cases with median duration of follow up 7.6 months (min-max: 1-41 months). Their mean age was 60.3 years (SD: 14.4 ). About $84 \%$ of them were males, $8.1 \%$ were diabetic, $9.7 \%$ were hypertensive, and $6.9 \%$ of them had asthma. 50 cases $(87.7 \%)$ had advanced tumor size (T3 or T4). At presentation, only 15 patients $(27.2 \%)$ had no lymph node metastases. Also, 47 patients $(83.9 \%)$ had metastases. For NSCLC, The most common pathological type was adenocarcinoma which was found in 26 cases (41.9\%), followed by 15 casas squamous cell carcinoma (24.2\%) and 14 cases SCLC (22.6). 38 cases $(80.9 \%)$ were presented at stage IV. For SCLC, 9 patients $(64.4 \%)$ were presented by extensive disease. 46 cases $(74.2 \%)$ express HHLA2 and about 21 positive cases (45.7\%) showed mild expression of HHLA2 compared to 20 cases $(43.2 \%)$ with moderate expression and only 5 cases $(8.1 \%)$ with high expression. EGFR was positive in 40 cases $(64.5 \%)$ of including 33 cases $(71.7 \%)$ of NSCLC compared to 5 cases $(35.7 \%)$ of SCLC, in addition to 2 cases of large cell neuroendocrine carcinoma $(\mathrm{p}=0.014)$.

\section{HHLA2 Expression in Relation to Other Clinicopathological Characteristics}

The association between HHLA2 tumour expression and various parameters of the patients is presented in Table 1 and Figure 1. There was no statistically significant associations between age or sex and the HHLA2 expression (p: 0.106, 0.709), respectively. Regarding the staging of the tumor, there was a significant association between HHLA 2 expression and metastasis. About $83 \%$ of patients presented with metastasis showed positive expression of HHLA2 compared to $44.4 \%$ in patients with no metastasis $(\mathrm{p}=0.024)$. Among cases with SCLC, HHLA2 expression was significantly higher in extensive stage $(80 \%)$, compared to $0 \%$ of limited stage, $(p=0.035)$. While, expression of HHLA2 was higher in stage IV of NSCLC cases $(86.5 \%)$, compared to stage II and III (44.4\%) $(\mathrm{p}=0.006)$. The pathological type was not associated with HHLA2 expression $(\mathrm{p}=0.488)$. Results show a significant mild positive correlation between expression of HHLA2 and EGFR markers $(\mathrm{r}=0.256, \mathrm{p}=0.045)$

Table 2 shows the association between the intensity of positive $H H L A 2$ expression and the clinicopathologic characteristics of the patients. None of the Clinicopathologic parameters of the patients was associated with the intensity of the expression of HHLA2. There was a nonsignificant correlation between the intensity of expression of HHLA2, and $E G F R(\mathrm{r}=0.307, \mathrm{p}=0.087)$

\section{Association between HHLA2 Expression and Survival}

A univariate analysis was done to assess the effect of 
Table 1. The Association of Clinicopathologic Characteristics with the Expression of HHLA2 (N=62).

\begin{tabular}{|c|c|c|c|c|}
\hline Clinicopathologic Characteristics & & $\begin{array}{c}\text { Negative } \\
\mathrm{N}(\%) \\
16(25.8)\end{array}$ & $\begin{array}{c}\text { Positive } \\
\text { N (\%) } \\
46(74.2)\end{array}$ & Significance \\
\hline Age (Ys) & Median (min-max) & $67(43-79)$ & $59(28-80)$ & $\mathrm{Z}=-1.6, \mathrm{p}=0.106^{\mathrm{a}}$ \\
\hline \multirow[t]{2}{*}{ Gender } & Male & $13(25)$ & $39(75)$ & $\mathrm{P}=0.709^{\mathrm{b}}$ \\
\hline & Female & $3(30)$ & $7(70)$ & \\
\hline \multirow[t]{2}{*}{ Tumor $(\mathrm{N}=57)$} & $\mathrm{T} 1 / \mathrm{T} 2$ & 0 & $7(100)$ & $\mathrm{P}=0.186$ \\
\hline & $\mathrm{T} 3 / \mathrm{T} 4$ & $13(26)$ & $37(74)$ & \\
\hline \multirow[t]{2}{*}{ Lymph nodes $(\mathrm{N}=55)$} & No & $3(20)$ & $12(80)$ & $\mathrm{P}=1^{\mathrm{b}}$ \\
\hline & $\mathrm{N} 2 / \mathrm{N} 3$ & $10(25)$ & $30(75)$ & \\
\hline \multirow[t]{2}{*}{ Metastasis $(\mathrm{N}=56)$} & M0 & $5(55.6)$ & $4(44.4)$ & $\mathrm{P}=0.024^{\mathrm{b}}$ \\
\hline & M1 & $8(17)$ & $39(83)$ & \\
\hline \multirow[t]{3}{*}{ Pathological types } & SCLC & $5(35.7)$ & $9(64.3)$ & $\mathrm{R}$ \\
\hline & NSCLC & $10(21.7)$ & $36(78.3)$ & $\mathrm{P}=0.309^{\mathrm{b}}$ \\
\hline & Large cell neuroendocrine carcinoma & $1(50)$ & $1(50)$ & $\mathrm{P}=1 \mathrm{~b}$ \\
\hline \multirow[t]{2}{*}{ Stage SCLC } & Limited & $5(100)$ & 0 & \\
\hline & Extensive & $1(11.1)$ & $8(88.9)$ & $\mathrm{P}=0.035^{\mathrm{b}}$ \\
\hline \multirow[t]{2}{*}{ Stage NSCLC } & II /III & $5(55.6)$ & $4(44.4)$ & $\mathrm{P}=0.006^{\mathrm{b}}$ \\
\hline & IV & $5(13.5)$ & $32(86.5)$ & \\
\hline \multirow[t]{2}{*}{ EGFR expression } & Negative & $9(40.9)$ & $13(59.1)$ & $\mathrm{R}=0.256^{*}, \mathrm{p}=0.045^{\mathrm{b}}$ \\
\hline & Positive & $7(17.5)$ & $33(82.5)$ & \\
\hline
\end{tabular}

a, Mann-Whitney U; ${ }^{\text {b }}$ Fisher's Exact Test; *, Spearman's rho

HHLA2 expression on survival. Kaplan-Meier survival curves were made, and then log-rank test was used.

Correlation of overall and progression-free survivals of cases and tumor expression of HHLA2 marker are presented in Table 3, and Figure 2. The mean OS time in cases with positive HHLA2 expression (17.6) was nearly half that of patients with negative expression of the markers (34.7). However, this difference was not statistically significant (p: 0.16). But, PFS of patients was significantly lower among the group with positive expression of HHLA2 (10.3) compared to the group with negative expression of HHLA2 (23.4) (p: 0.010).

\section{Discussion}

Lung cancer remains the world's major cause of

Table 2. The Association of Clinicopathologic Characteristics with the Intensity of Expression of HHLA2 (N=46).

\begin{tabular}{|c|c|c|c|c|}
\hline \multicolumn{2}{|c|}{ Clinicopathologic Characteristics } & \multirow{2}{*}{$\begin{array}{c}\text { Mild } \\
\text { N (\%) } \\
21(45.7) \\
58(31-72)\end{array}$} & \multirow{2}{*}{$\begin{array}{c}\text { Moderate/ Strong } \\
\mathrm{N}(\%) \\
25(54.3) \\
60.5(28-80)\end{array}$} & \multirow[t]{2}{*}{ Significance } \\
\hline Age (Ys) & Median (min-max) & & & \\
\hline \multirow[t]{2}{*}{ Gender } & Male & $20(51.3)$ & 19 (48.7) & $\mathrm{P}=0.106^{\mathrm{b}}$ \\
\hline & Female & $1(14.3)$ & $6(85.7)$ & \\
\hline \multirow[t]{2}{*}{ Tumor $(\mathrm{N}=44)$} & $\mathrm{T} 1 / \mathrm{T} 2$ & $2(28.6)$ & $5(71.4)$ & $\mathrm{P}=0.428^{b}$ \\
\hline & $\mathrm{T} 3 / \mathrm{T} 4$ & $18(48.6)$ & $19(51.4)$ & \\
\hline \multirow[t]{2}{*}{ Lymph nodes $(\mathrm{N}=42)$} & No & $4(33.3)$ & $8(66.7)$ & $\mathrm{X}^{2}=0.96, \mathrm{p}=0.327$ \\
\hline & $\mathrm{N} 2 / \mathrm{N} 3$ & $15(50)$ & $15(50)$ & \\
\hline \multirow[t]{2}{*}{ Metastasis $(\mathrm{N}=43)$} & M0 & $1(25)$ & $3(75)$ & $\mathrm{P}=0.610^{\mathrm{b}}$ \\
\hline & M1 & $19(48.7)$ & $20(51.3)$ & \\
\hline \multirow[t]{3}{*}{ Pathological types } & SCLC & $4(44.4)$ & $5(55.6)$ & $\mathrm{R}$ \\
\hline & NSCLC & $16(44.4)$ & $20(55.6)$ & $\mathrm{p}=1^{\mathrm{b}}$ \\
\hline & Large cell neuroendocrine carcinoma & $1(100)$ & 0 & $\mathrm{P}=1^{\mathrm{b}}$ \\
\hline \multirow[t]{2}{*}{ Stage NSCLC } & II/ III & $1(25)$ & $3(75)$ & $\mathrm{P}=0.613^{\mathrm{b}}$ \\
\hline & IV & $15(46.9)$ & $17(53.1)$ & \\
\hline \multirow[t]{2}{*}{ Positive EGFR } & Mild & $8(61.5)$ & $5(38.5)$ & $\mathrm{r}=0.307^{*}, \mathrm{p}=0.087$ \\
\hline & Moderate/strong & $13(39.4)$ & $20(60.6)$ & \\
\hline
\end{tabular}

a, Mann-Whitney U; b, Fisher's Exact Test; *, Spearman's rho 
Table 3. The Analysis of Survival in Relation to Tumor Expression of HHLA (Kaplan-Meier Test).

\begin{tabular}{llccccc}
\hline & Total N & N of events & $\begin{array}{c}\text { Censored } \\
\text { N (\%) }\end{array}$ & $\begin{array}{c}\text { Survival time } \\
\text { Mean }(95 \% \mathrm{CI})\end{array}$ & $\mathrm{p}$ \\
\hline Overall Survival & Negative & 12 & 1 & $11(91.7)$ & $34.7(24.5-44.8)$ & 0.16 \\
& Positive & 43 & 8 & $35(81.4)$ & $17.6(14.9-20.3)$ & \\
\multirow{2}{*}{ Progression Free Survival } & Negative & 4 & 4 & $8(55.3)$ & $23.4(10.6-36.4)$ & 0.01 \\
& Positive & 43 & 17 & $29(58)$ & $10.3(9.5-18.1)$ & \\
\hline
\end{tabular}

*, Log Rank (Mantel-Cox)
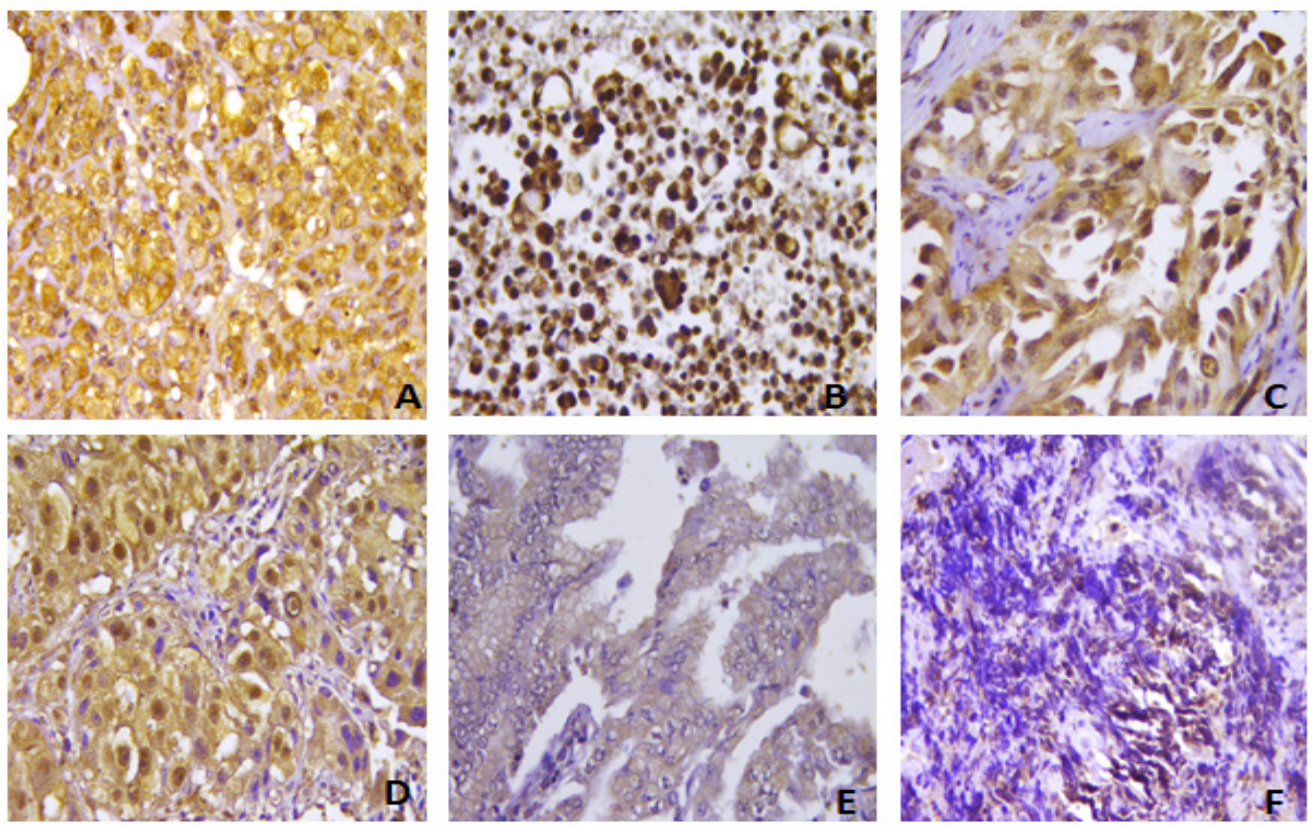

Figure 1. Immunohistochemical Staining of HHLA2 in Different Cases of Lung Carcinoma: Strong expression in a case of squamous cell carcinoma (A) and in a case of papillary adenocarcinoma (B). Moderate HHLA2 expression in a case of acinar adenocarcino

mortality, and is one of the most frequently diagnosed cancers (Lin, 2019). Tumor immune evasion is one of the hallmarks of malignancy, and expression of the B7 family of ICPs Immune checkpoint molecules ( $P D-L 1, P D-L 2$, $B 7-H 3, B 7 x$ and HHLA2) is one mechanism of immune evasion by tumors. These molecules could suppress of T-cell function and proliferation and subsequently cytokine production (Flajnik et al., 2012; Zhao et al., 2013; Zhu et al., 2013; Janakiram et al., 2015b; Janakiram et al., 2017; Cheng et al., 2017). Given its emerging role
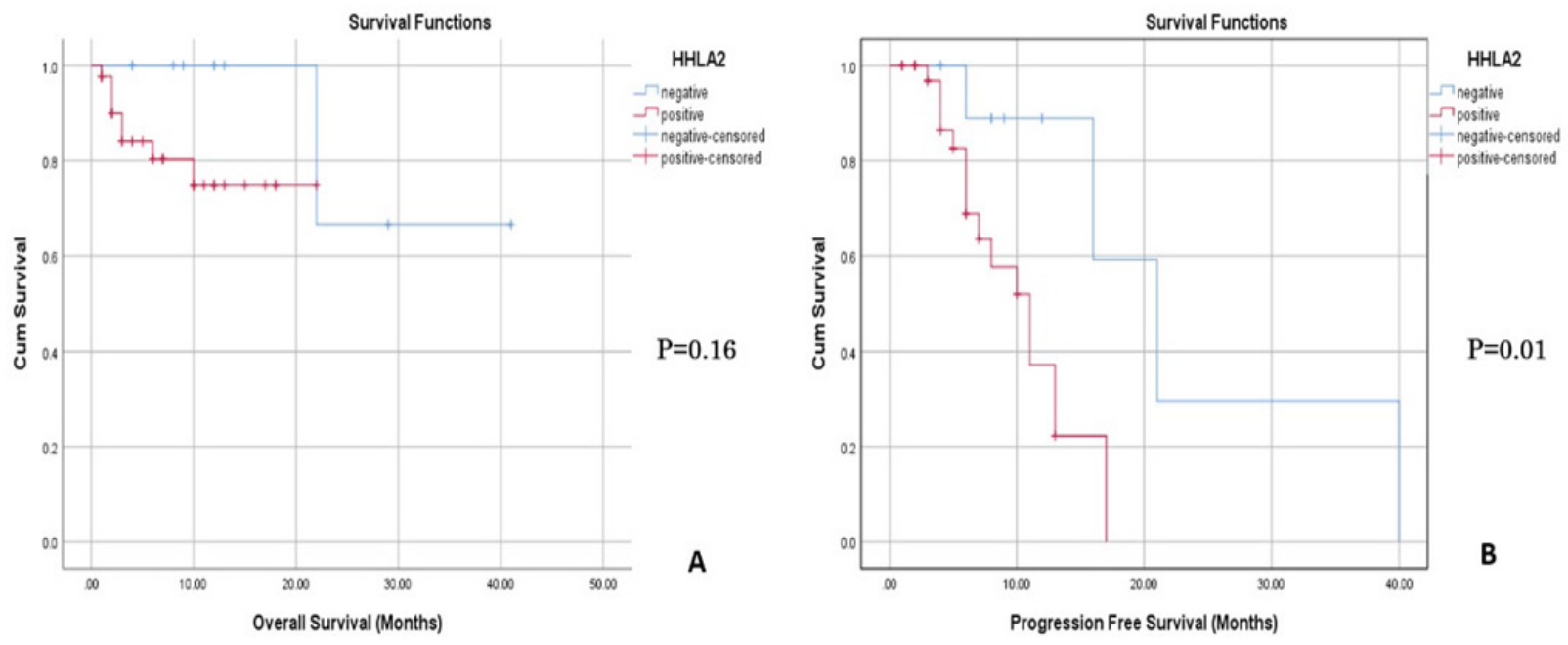

Figure 2. Analysis of Survival in Relation to Tumor Expression of HHLA2: (A) Overall survival (OS) and HHLA2: Positive expression was lower than patients with negative HHLA2 (p: 0.16) (B) Progression free survival (PFS) and HHLA2: PFS is lower among 
of suppressing tumor immune responses with possible different immune evasion mechanisms from PD-1/PD-L1 pathways (Zang et al., 2010; Janakiram et al., 2012), there has been a great interest for exploration of HHLA2 as a therapeutic immune target (Jeon et al., 2014; Janakiram et al., 2015b). Targeting these interactions through immunomodulation drugs has remarkable clinical success in several cancers (Inal et al., 2015).

In the current study, we examined immunohistochemical HHLA2 expression in lung carcinoma specimens and its correlation to clinicopathological features. We studied HHLA 2 expression in NSCLC (adenocarcinoma, squamous cell carcinoma, and large cell undifferentiated carcinoma) and SCLC. HHLA2 expression was found in $74 \%$ of the patients with tendency towards increased expression in NSCLC (78.3\% of the positive cases). Similar to our results, Cheng et al., (2017) firstly reported high expression of HHLA2 in lung cancer. It was positive in $71 \%$ of patients in a cohort study and also was expressed in two thirds (66\%) of NSCLC cases (Cheng et al., 2017). In a more recent study by cheng et al, 2018, HHLA2 was positive in most of lung cancer specimens, including $61 \%$ of neoplasms in the discovery set and $64 \%$ in the validation cohort (Cheng et al., 2018).

In our study, there were no statistically significant associations between age or gender and the HHLA2 expression. This is consistent with what was previously reported in a study done by cheng et al., (2017) (Cheng et al., 2017). Also, we found no significant relationship between HHLA2 expression and both tumor and lymph node stages. Similarly HHLA2 expression was not correlated to lymph node status in lung cancer (Cheng et al., 2017). But in triple-negative breast cancer (TNBC), overexpressed HHLA2 was linked to lymph node positivity (Janakiram et al., 2015a).

However, a statistically significant association was detected between HHLA2 expression and both tumor staging and metastasis. Our results found that HHLA2 expression was higher in metastatic, stage IV disease than stage II and III disease. On the contrary, no association between HHLA2 expression and various stages of lung cancer was revealed in the study by Cheng et al., (2017). But in TNBC, overexpressed HHLA2 was linked to advanced stage of cancer at diagnosis, and also was linked to a high recurrence risk (Janakiram et al., 2015a). Also, HHLA2 expression in osteosarcoma was reported to be associated with metastases (Koirala et al., 2016).

In our study, EGFR was significantly expressed in NSCLC compared to SCLC ( $\mathrm{p}=0.014)$. It is in agreement with previous studies which confirmed that EGFR mutations were one of the commonest oncogenic changes in NSCLC. Lung carcinoma cases with such mutations show distinct pathogenesis, clinical manifestations and disease course (Janakiram et al., 2016).

As regards the Association of HHLA2 and EGFR, results revealed that $E G F R$ expression was significantly correlated with HHLA2 expression. Positive HHLA2 staining was found in $82.5 \%$ of cases with positive $E G F R$ expression $(\mathrm{P}=0.045)$. This finding is in line with previous studies which reported that $E G F R$ mutation was significantly correlated with overexpression of HHLA2.
This indicates that HHLA2 is a potentially novel target for lung cancer immunotherapy, especially in patients with $E G F R$ expression. Also, this is particularly significant because HHLA2 expression may assist identification of a group of patients who might do poorly in spite of tyrosine kinase inhibitors (TKIs) for EGFR-mutated tumours (Janakiram et al., 2016; Cheng et al., 2017; Cheng et al., 2018).

Few studies were done on the prognostic value of HHLA2 in lung cancer. In our study, HHLA2 overexpression with decreased survival was frequent. However this was not statistically significant with OS $(\mathrm{p}=0.16)$. On the other side, a significant relation was detected with PFS $(p=0.01)$, where patients with positive expression had shorter OS and PFS in comparison to patients with negative expression ( 17.6 vs $34.7 \mathrm{~m}, 10.3$ vs $23.4 \mathrm{~m}$ ) respectively.

Our results are in line with a previous study which found that tumor HHLA2 expression was significantly correlated with OS of NSCLC. In EGFR mutated tumors, there was a trend towards $H H L A 2$ overexpression with reduced OS $(p=0.19)$. However, this was not statistically significant (Cheng et al., 2017).

Many studies have reported that HHLA2 was a negative indicator in many malignancies as colon, lung and pancreatic malignancies (Cheng et al., 2017; Yan et al., 2019; Zhang et al., 2020; Zhu and Dong, 2018). The poor prognostic value of HHLA2 was also reported in clear cell RCC, osteosarcoma and TNBC (Janakiram et al., 2015a; Koirala et al., 2016; Chen et al., 2018; Chen et al., 2019). However, high HHLA2 expression was linked to better postsurgical prognosis in pancreatic and ampullary malignancies when utilizing a different anti-HHLA2 antibody clone for IHC (Boor et al., 2020).

This indicates that the prognostic and clinical significance of HHLA2 expression in different tumors might be complex and influenced by several factors (Chen et al., 2018). Novel immunotherapeutic approaches targeting HHLA2 are under active development and have demonstrated hopeful results in many studies on other cancers. The wide expression of HHLA2 in lung cancer implicates the therapeutic potential of targeting those immune markers also in lung cancer (Dangaj et al., 2013; Janakiram et al., 2017).

Our study had some limitations since it is a retrospective one and other etiological factors including smoking and therapeutic factors following relapse could affect OS. Other factors influencing HHLA2 expression such as T- cell subsets, expression of other co-inhibitory molecules as $P D-L 1, B 7-H 3$ and $B 7 x$ were not assessed. Further research is required to determine the biological significance and detect mechanisms of high HHLA2 expression in lung cancer and clarify its contributions in tumor immune escape.

In conclusions, This study suggests that recently discovered, HHLA2 is over expressed in lung cancer associating with higher stage. It is also correlated with EGFR overexpression. HHLA2 could serve as a predictor of progression and distant metastasis. Also, it has potential to be effective immune target in lung cancer immunotherapy such as checkpoint blockade and 
antibody-drug conjugate treatment.

\section{Author Contribution Statement}

Author 1: Concepts, Design, Literature search, Data analysis, Manuscript editing\& review Authors 2, 3, 6: Clinical Studies and Data acquisition. Author 4: Data \& Statistical analysis and manuscript review.

MF put the research design, did the literature search, performed the histological examination, analyzed data, wrote \& reviewed the manuscript. EI performed the histological examination. TE, MA, AE \& HA acquired the clinical data and did the clinical studies. HA wrote and reviewed the manuscript. All authors read and approved the final manuscript.

\section{Funding Sources}

No funding was provided for this research.

\section{Disclosure Statement}

The author has no conflicts of interest to declare.

\section{Statement of Ethics}

The work was being performed in agreement with the rules in the Declaration of Helsinki and was approved by Institutional Research Board (IRB) of Faculty of Medicine, Mansoura University (R.20.09.1026).

This research was not approved by any scientific body and also was not a part of any approved student thesis.

\section{References}

Boor PPC, Sideras K, Biermann K, et al (2020). HHLA2 is expressed in pancreatic and ampullary cancers and increased expression is associated with better post-surgical prognosis. Br J Cancer, 122, 1211-8.

Chang Y-C, Hsu P-C, Li S-H, et al (2019). The prevalence of PD-L1 expression in lung cancer. Clin Oncol, 4, 1591.

Chen D, Chen W, Xu Y, et al (2018). Upregulated immune checkpoint HHLA2 in clear cell renal cell carcinoma: a novel prognostic biomarker and potential therapeutic target. $J$ Med Genet, 56, 43-9.

Chen L, Zhu D, Feng J, et al (2019). Overexpression of HHLA2 in human clear cell renal cell carcinoma is significantly associated with poor survival of the patients. Cancer Cell Int, 19, https://doi.org/10.1186/s12935-019-0813-2.

Chen Z, Fillmore CM, Hammerman PS, Kim CF, Wong K-K (2014). Non-small-cell lung cancers: a heterogeneous set of diseases. Nat Rev Cancer, 14, 535-46.

Cheng H, Borczuk A, Janakiram M, et al (2018). Wide expression and significance of alternative immune checkpoint molecules, B7x and HHLA2, in PD-L1-negative human lung cancers. Clin Cancer Res, 24, 1954-64.

Cheng H, Janakiram M, Borczuk A, et al (2017). HHLA2, a new immune checkpoint member of the B7 family, is widely expressed in human lung cancer and associated with EGFR mutational status. Clin Cancer Res, 23, 825-32.

Dangaj D, Lanitis E, Zhao A, et al (2013). Novel recombinant human B7-H4 antibodies overcome tumoral immune escape to potentiate T-cell antitumor responses. Cancer Res, 73, 4820-9.

Flajnik MF, Tlapakova T, Criscitiello MF, Krylov V, Ohta Y (2012). Evolution of the B7 family: co-evolution of B7H6 and NKp30, identification of a new B7 family member,
B7H7, and of B7's historical relationship with the MHC. Immunogenetics, 64, 571-90.

Ibrahim AS, Khaled HM, Mikhail NNH, Baraka H, Kamel H (2014). Cancer Incidence in Egypt: Results of the National Population-Based Cancer Registry Program. J Cancer Epidemiol, 2014, 1-18.

Inal C, Yilmaz E, Piperdi B, Perez-Soler R, Cheng H (2015). Emerging treatment for advanced lung cancer withEGFRmutation. Expert Opin Emerg Drugs, 20, 597-612.

Janakiram M, Abadi YM, Sparano JA, Zang X (2012). T cell coinhibition and immunotherapy in human breast cancer. Discov Med, 14, 229.

Janakiram M, Chinai JM, Fineberg S, et al (2015a). Expression, clinical significance, and receptor identification of the newest B7 family member HHLA2 protein. Clin Cancer Res, 21, 2359-66.

Janakiram M, Chinai JM, Zhao A, Sparano JA, Zang X (2015b). HHLA2 and TMIGD2: new immunotherapeutic targets of the B7 and CD28 families. Oncoimmunology, 4, e1026534.

Janakiram M, Pareek V, Cheng H, Narasimhulu DM, Zang X (2016). Immune checkpoint blockade in human cancer therapy: lung cancer and hematologic malignancies. Immunotherapy, 8, 809-19.

Janakiram M, Shah UA, Liu W, et al (2017). The third group of the B7-CD28 immune checkpoint family: HHLA2, TMIGD2, B7x, and B7-H3. Immunol Rev, 276, 26-39.

Jeon H, Vigdorovich V, Garrett-Thomson SC, et al (2014). Structure and cancer immunotherapy of the B7 family member B7x. Cell Rep, 9, 1089-98.

Koirala P, Roth ME, Gill J, et al (2016). HHLA2, a member of the B7 family, is expressed in human osteosarcoma and is associated with metastases and worse survival. Sci Rep, 6, https://doi.org/10.1038/srep31154.

Lin C-C (2019). Challenges of the phase I drug development in non-small cell lung cancer. Chin Clin Oncol, 8, 25.

Petersen I, Dietel M, Geilenkeuser WJ, et al (2017). EGFR immunohistochemistry as biomarker for antibody-based therapy of squamous NSCLC - Experience from the first ring trial of the German Quality Assurance Initiative for Pathology (QuIP ${ }^{\circledR}$ ). Pathol Res Pract, 213, 1530-5.

Torre LA, Lindsey A, Rebecca L (2016). Lung cancer Stat. $A d v$ Exp Med Biol, 893, 1-19.

Yan H, Qiu W, Koehne de Gonzalez AK, et al (2019). HHLA2 is a novel immune checkpoint protein in pancreatic ductal adenocarcinoma and predicts post-surgical survival. Cancer Lett, 442, 333-40.

Zago G, Muller M, Van Den Heuvel M, Baas P (2016). New targeted treatments for non-small-cell lung cancer- Role of nivolumab. Biol Targets Ther, https://doi.org/10.2147/ BTT.S87878.

Zang X, Sullivan PS, Soslow RA, et al (2010). Tumor associated endothelial expression of B7-H3 predicts survival in ovarian carcinomas. Mod Pathol, 23, 1104-12.

Zhang C, Leighl NB, Wu Y-L, Zhong W-Z (2019). Emerging therapies for non-small cell lung cancer. J Hematol Oncol, 12, https://doi.org/10.1186/s13045-019-0731-8.

Zhang Z, Liu J, Zhang C, et al (2020). Over-expression and prognostic significance of HHLA2, a new immune checkpoint molecule, in human clear cell renal cell carcinoma. Front Cell Dev Biol, 8. https://doi.org/10.3389/ fcell.2020.00280

Zhao R, Chinai JM, Buhl S, et al (2013). HHLA2 is a member of the B7 family and inhibits human CD4 and CD8 T-cell function. Proc Natl Acad Sci U S A, 110, 9879-84.

Zhu Y, Yao S, Iliopoulou BP, et al (2013). B7-H5 costimulates human T cells via CD28H. Nat Commun, 4. 
Zhu Z, Dong W (2018). Overexpression of HHLA2, a member of the B7 family, is associated with worse survival in human colorectal carcinoma. Onco Targets Ther, 11, 1563-70.

\section{(ब) $(1) \otimes$}

This work is licensed under a Creative Commons AttributionNon Commercial 4.0 International License. 